\title{
Interference with avoidance behavior as a function of qualitative properties of inescapable shocks
}

\author{
J. A. LAWRY \\ University of Minnesota, Minneapolis, Minnesota 55455 \\ V. LUPO \\ University of Notre Dame, Notre Dame, Indiana 46556
}

J. J. OVERMIER, J. KOCHEVAR, and K. L. HOLLIS

University of Minnesota, Minneapolis, Minnesota 55455

and

D. C. ANDERSON
University of Notre Dame, Notre Dame, Indiana 46556

\begin{abstract}
Temporal form (continuous vs. pulsating) and shock source (alternating current vs. direct current) were factorially combined to produce four shock treatments. The effects of inescapable presentations of these stimuli on subsequent avoidance response acquisition were measured in dogs (Experiment 1) and in rats (Experiment 2) and revealed an interaction of shock variables. Initially, all groups that received ac shock showed impaired performance for the pulsating and continuous shock conditions; groups that received dc continuous shock were also impaired, while those that received dc pulsating shock were not. While this pattern of interference persisted for dogs, it was transient in rats, with only the ac continuous-shock group continuing to be impaired. Mean avoidance performance were positively related to mean activity levels during inescapable shocks for the dc shock groups but not for the ac shock groups.
\end{abstract}

Exposing dogs restrained in a Pavlovian harness to a series of inescapable shocks has been shown to produce marked interference with later acquisition of escape-avoidance responding (Overmier \& Seligman, 1967). To account for their findings, Overmier and Seligman hypothesized that the subject learns that shock termination is independent of its behavior, and the phrase "learned helplessness" was adopted to characterize the nature of the proposed interference mechanism. Later, "learned helplessness" was extended by Maier, Seligman, and Solomon (1969) as a formal hypothesis to include the notion that independence between the dog's behavior and features of the shock stimulus (e.g., onset, termination,

This research was supported by the following grants: $\mathrm{MH}-13,588$ to J. Bruce Overmier, HD-1554 to D. C. Anderson, HD-01136 and BNS-7.5-03816 to the Center for Research in Human Learning, and grants from the Graduate School of the University of Minnesota to the Center for Research in Human Learning and to J. B. Overmier. The authors are grateful to Raymond $O$. Collier, $\mathrm{Jr}$, for his guidance in the statistical analysis of the data. Requests for reprints should be sent to J. B. Overmier, Psychology, 75 East River Road, University of Minnesota, Minneapolis, Minnesota 55455 intensity, etc.) reduces the incentive to respond in the presence of shock.

This interfering effect of inescapable shock on subsequent shuttle performance (which will be referred to here as the "interference phenomenon") has since led to substantial research and further theorizing (see Maier \& Seligman, 1976, for a review), with much of the current work utilizing rats as subjects. There have, however, been numerous difficulties in reliably producing the interference phenomenon in rats (Freda \& Klein, 1976; Weiss, Krieckhaus, \& Conte, 1968). Anderson, Lupo, Cunningham, and Madden (Note 1) reported that inescapable shocks of different temporal forms (i.e., pulsating and continuous), markedly influenced whether or not rats showed subsequent impairment on an escape-avoidance task. They also noted that shocks of different temporal forms were associated with different activity patterns during the inescapable shock treatment. Rats that received continuous shock throughout the 5-sec presentations showed little gross motor activity during the inescapable shock treatment and considerable impairment on the escape-avoidance task, while rats that received pulsating shock (i.e., $50 \mathrm{msec}$ 
on-off times) during the 5-sec presentations showed persistently high levels of activity and little or no impairment on the escape-avoidance task. Anderson et al. (Note 1) hypothesized that the activity during the inescapable shock treatment might reflect the acquisition of certain behaviors which are compatible (as in the case of the pulsating shock group) or incompatible (as in the case of the continuous shock group) with the behavior required during subsequent tests and thus determine whether or not interference with escape-avoidance performance will be observed. This importance of the temporal form of inescapable shock suggests that "inescapability" of shock may not be a sufficient factor for producing subsequent interference with avoidance behavior.

A survey of the "learned helplessness" literature in dogs indicates that continuous inescapable shocks have been used in all cases, thereby not allowing for an evaluation of the potential importance of the temporal form. In addition, these studies, taken as a. group, also differ from Anderson's with respect to the type of shock source used to produce the inescapable shocks: an alternating current (ac) shock source was used in all of the dog studies, while Anderson and his colleagues have always used a direct current (dc) shock source.

It is well known that temporal form is an important modulator of behavior; D'Amato and Fazzaro (1966) showed that pulsated shocks facilitate avoidance learning in rats. Similarly, ac and dc shock stimuli can have different behavioral effects. For example, Campbell and Teghtsoonian (1958) reported that ac and $\mathrm{dc}$ shock sources yield different aversion thresholds as well as different activity levels. Alternating and dc shocks of functionally similar intensities also have been demonstrated to have differential effects on other behavioral phenomena, including tonic immobility in chickens (Nash \& Gallup, 1975) and shock-induced fighting in rats (Follick \& Knutson, 1974).

Taken together, the behavioral differences that are related to the temporal form of shock and type of shock source raise a number of methodological questions regarding the study of interference with escapeavoidance responding following exposure to inescapable shock. Do ac and dc inescapable shocks produce equivalent interference effects? Do pulsated and continuous shocks produce differential effects, as reported by Anderson et al. (Note 1) regardless of shock source? And finally, is the pattern found in comparing shock sources and temporal form consistent for rats and dogs? The following two experiments were conducted to examine these methodological questions.

\section{EXPERIMENT 1}

This study, conducted at the University of Minnesota laboratories with dogs as subjects, used a 2 by 2 factorial design to determine the degree of interference with subsequent avoidance learning as a function of type of shock source (ac vs. dc) and temporal form (constant vs. pulsating) used in the inescapable shock treatment.

In general, the procedures employed during the administration of the inescapable shocks and the test phase were quite similar to those used by Overmier and Seligman (1967) in their original demonstration of the interference effect.

\section{Method}

\section{Subjects and Apparatus}

The subjects were 36 adult random-source dogs, with shoulder heights of 35 to $48 \mathrm{~cm}$, obtained from the University of Minnesota Animal Hospital. They were maintained in individual cages and given free access to food and water throughout the experiment.

The apparatus in which inescapable shocks were presented consisted of a rubberized cloth hammock suspended from a metal frame located inside a large, white, sound-reducing cubicle. The hammock was constructed so that the dog's legs hung down below the body through four holes and could be secured in that position. A leash from the ceiling was attached to a neck collar to prevent escape from the apparatus. Stainless steel plate electrodes $(7 \times 12 \mathrm{~cm})$, coated with electrode paste, were taped to the footpads of the dog's hind feet. Either of two electric shock sources delivered shocks to the electrodes. One was a $600-\mathrm{V}$ ac variable transformer, with current applied through a resistance of $20 \mathrm{k}$. The shock level was adjusted for each dog on the first shock presentation to $5.0 \mathrm{~mA}$. The second shock source was a constant current, tube regulated, dc device after Campbell and Teghtsoonian (1958), set at $5.0 \mathrm{~mA}$. For those groups receiving pulsating shock, a pulse-stream generator interrupted the shock at a rate of 10 times $/ \mathrm{sec}$, with equal on-off intervals.

An Alton ultrasonic motion detector (Model 5) was used to record movements of the subjects during the 5-sec inescapable shock periods. The transmitting and receiving transducers of this device were located $23 \mathrm{~cm}$ apart, center to center, and on a ledge at a height equal to the subject's back while resting in the harness and approximately $\mathbf{4 0} \mathrm{cm}$ from the subject's left side. The receiving transducer was positioned to be perpendicular to the head and shoulder alignment of the subject. The sensitivity adjustment, (.8) was such that all visible gross body movements were readily recorded.

White noise of approximately $70 \mathrm{~dB}$ was presented in the cubicle throughout the inescapable shock treatment session. Electromechanical recording and programming equipment was located outside the cubicle.

Testing was conducted in a two-way shuttlebox consisting of two black compartments separated by an adjustable barrier. The barrier was adjusted to the shoulder height of each subject just prior to testing. Each compartment was $114 \mathrm{~cm}$ long $\times 61 \mathrm{~cm}$ wide $\times 102 \mathrm{~cm}$ high. Illumination was provided by one $15-\mathrm{W}$ and one 150.W lamp located above a wire mesh ceiling in each of the compartments. The CS consisted of turning off the 150-W lamp located above each compartment, which produced a sharp decrease in the level of illumination. Electric shock was delivered to the shuttlebox from a $0-600-\mathrm{V}$ ac variable transformer through a series resistance of $50 \mathrm{k} \Omega$. The shock was set at $4.5 \mathrm{~mA}$ and administered via a grid floor consisting of $3.2 \mathrm{~cm}$ flat aluminum bars placed $1.6 \mathrm{~cm}$ apart. A commutator shifted polarity patterns six times/sec. Whenever a subject crossed the barrier, photocell beams were interrupted, a response was automatically recorded, and the ongoing trial was terminated. Latencies of barrier crossings were measured to the nearest $.1 \mathrm{sec}$ and recorded by a printout counter. Stimulus presentations and temporal contingencies were controlled by automatic relay circuitry housed in an adjoining room. White noise of approximately $70 \mathrm{~dB}$ was present in the shuttlebox throughout testing. 


\section{Procedure}

Inescapable shock treatment. After being secured in the harness with electrodes in place, each subject was give 5 min to adapt to the apparatus before the shock treatment began. Groups AC-C, AC-P, DC-C, and DC-P each received 645 -sec unsignaled shocks programmed to be inescapable. The intershock interval (ISI) varied from 60 to $120 \mathrm{sec}$, with an average of $90 \mathrm{sec}$. Groups AC-C and $A C$-P received shock from the ac shock source, while Groups DC-C and DC-P received shock from the dc shock source. Groups AC-C and DC-C received $5 \mathrm{sec}$ of continuous shock, while Groups AC-P and DC-P received $5 \mathrm{sec}$ of pulsating shock. Group NS was restrained for an equivalent period of time in the harness, but no shocks were delivered. Group NT received no treatment prior to testing in the shuttlebox.

Testing for interference. Two tests were conducted. The first was approximately $24 \mathrm{~h}$ following pretreatment and the second was approximately $48 \mathrm{~h}$ later. Each test session consisted of 20 trials of instrumental escape-avoidance training in the shuttlebox by the traditional method of emergence (Solomon \& Brush, 1956). The onset of the CS (dimmed illumination) initiated each trial. If the subject crossed the barrier within $10 \mathrm{sec}$ of CS onset, the CS was terminated and shock was not presented (avoidance). If the dog failed to jump within $10 \mathrm{sec}$ of CS onset, a $4.5-\mathrm{mA}$ shock was presented and remained on until the subject crossed the barrier, terminating both the CS and the shock (escape). If no response occurred within $60 \mathrm{sec}$, the trial was automatically terminated and a latency of $60 \mathrm{sec}$ was recorded. If no responses were made in any of the successive five-trial blocks, the animal was moved to the other compartment. Intertrial intervals (ITI) ranged from 60 to $120 \mathrm{sec}$, with an average of $90 \mathrm{sec}$.

\section{Results}

Figure 1 shows the mean activity rates during shock presentation across blocks of eight trials for each shock treatment group. Due to difficulties with the transducers on the motion detection device at the start of the experiment, activity data were collected and analyzed for only the last five dogs within each shock treatment group.

The mean activity values for the AC-C, AC-P, DC-C, and DC-P groups were 7.6, 13.4, 3.3, and 10.3 , respectively. a Source by Form by Blocks ANOVA was performed on the mean trial activity counts taken for eight-trial blocks of the inescapable shock presentations. Temporal form of the inescapable shocks had a pronounced effect on our dogs' activities; the pulsating shock groups showed more movement than the continuous shock groups, $F(1,16)$ $=17.66, \mathrm{p}<.001$. Shock source was also a significant factor influencing activity rates, $F(1,16)=5.86$, $p<.05$. While overall the ac groups had somewhat higher rates than did the corresponding dc groups, the differential effects of shock source were less apparent than those of form.

Pairwise comparisons revealed that the differences between the two continuous shock groups and between the two pulsating groups were not significant, $t(16)=1.98$ and 1.45 , respectively, $p>.05$ for each. However, the difference between the two ac shock groups was significant, $\mathrm{g}(16)=2.71, \mathrm{p}<.05$, as was the difference between the two dc shock

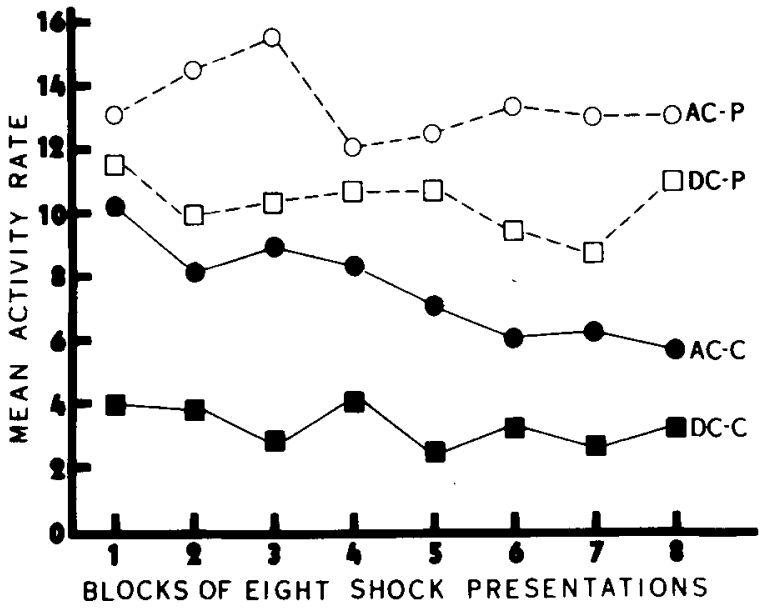

Figure 1. Mean activity rates across blocks of eight inescapable shock presentations for each experimental group in Experiment 1. Group treatments differed with respect to shock source (i.e., $\mathrm{AC}$ or DC) and temporal form of shock (i.e., Continuous or Pulsating). Data are presented for five dogs in each group, and each point represents the mean of 40 shock presentations ( 5 dogs $\times 8$ trials).

groups, $t(16)=3.24, p<.01$. Although there was some change in activity across blocks, resulting in a significant main effect for blocks $F(7,112)=2.75$, $\mathrm{p}<.05$, the relative ordering of the groups remained fixed, and there were no significant interactions between blocks and the shock source and form factors.

\section{Test Data}

Figures 2 and 3 show the mean latencies and mean number of failures to escape for each group across

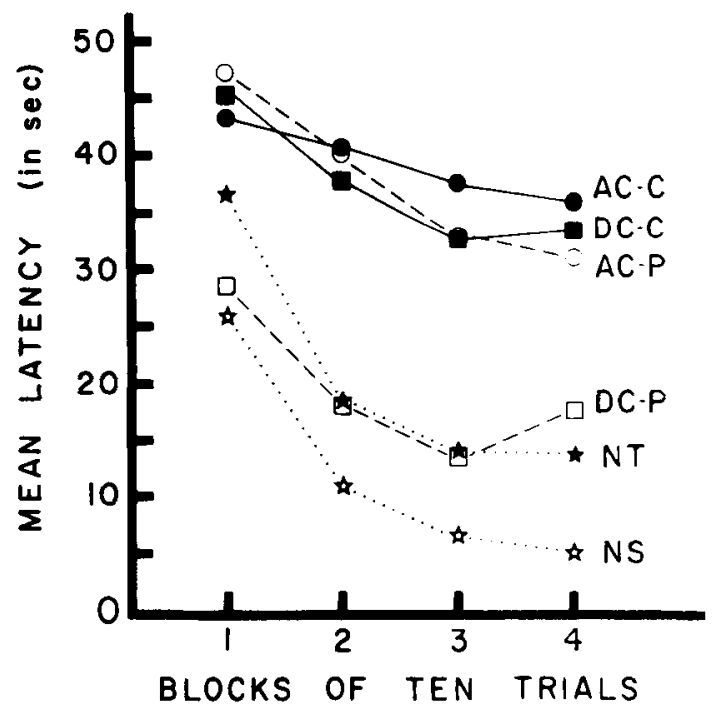

Figure 2. Mean shuttle response latency from $\mathrm{CS}$ onset for each group in Experiment 1 across blocks of 10 signaled escapeavoidance trials. Two blocks were presented on each of two test sessions separated by $48 \mathrm{~h}$. Groups differ with respect to inescapable shock treatment received prior to testing. 


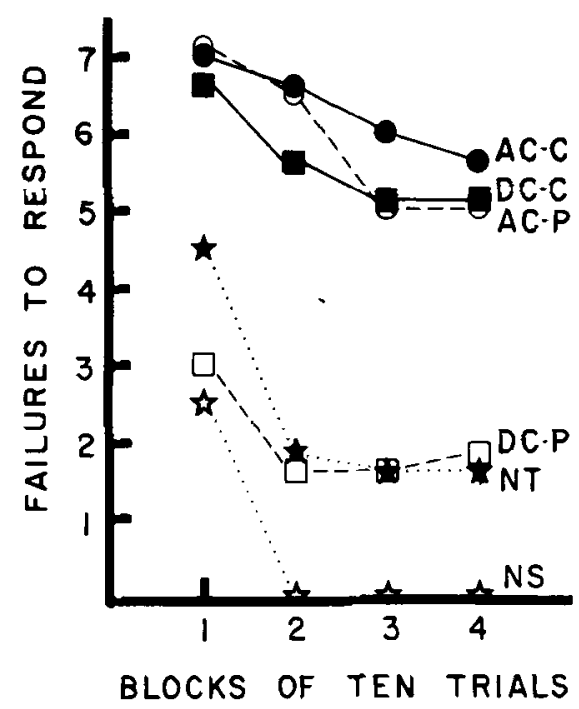

Figure 3. The mean number of failures to respond for each group in Experiment 1 across blocks of 10 signaled escapeavoidance trials. Twenty trials were presented on each of two test sessions. Groups differed with respect to inescapable shock treatment received prior to testing.

the four blocks of 10 test trials. Due to certain characteristics of the data (e.g., arbitrarily restricted range, bimodality of some distributions, etc.), the distribution-free randomization test procedures were applied (see Edgington, 1969a). Probabilities for the obtained group comparisons were based on 1,000 randomizations of the population of observed scores (Edgington, 1969b). The first analysis used the 36 individual subject scores randomly assigned, independent or original subject designation, to six pseudogroups corresponding to the six treatment conditions used in the experiment. The statistic of interest is the probability of obtaining the empirically observed difference score, given the population of possible difference scores for the comparison of interest derived from the randomizations. A second set of analyses involved only the shock groups; in these, the scores were randomized into four pseudogroups corresponding to the 2 by 2 factorial arrangements of the shock treatments. All comparisons between the controls and the experimental shock groups were one-tailed, while comparisons made among the experimental shock groups were twotailed. Results of approximate randomization tests such as these are isomorphic, with more traditional F-ratios under permutation (Edgington, 1969a; see also Fisher, 1935, on $F_{t}$ ).

Overmier and Seligman (1969) used only 10 trials to evaluate interference in the acquisition of escapeavoidance responding. We therefore decided first to evaluate separately the first 10 test trials of this experiment to provide a comparison with the original demonstration of this phenomenon in dogs.
Comparisons between two control groups with respect to both latencies of response and number of failures to respond did not reveal any impairment due to restraint, and in fact the restrained group, NS, had somewhat shorter latencies and fewer failures than did the unrestrained group, NT. For all subsequent comparisons with the shocked groups, the two control groups were combined. The combined shock groups were impaired relative to the controls, and this was evident by their longer mean latency, $\mathrm{p}<.001$, and greater number of failures to respond on the first 10 test trials, $p<.05$. The pattern of interference, as reflected by both indices, was influenced by both shock source and temporal form with these factors interacting, $p<.05$. Therefore, each shock group was separately compared with the controls to evaluate whether each of the sourceform treatment combinations resulted in interference. Groups AC-C, AC-P, and DC-C each had longer mean latencies, $p<.001$ for each, and more failures to respond, $\mathrm{p}<.05$ for each, than did the controls. There were no reliable differences among these three shock groups on either response measure. Group DC-P, on the other hand, showed no such impairment in learning, as its mean latency and number of failures to respond were statistically indistinguishable from the controls. The DC-P group did, however, have a significantly shorter mean latency than either AC-P or DC-C, $\mathrm{p}<.001$ for each, but no significant differences were found between it and any of the other shock groups for the number of failures to respond, reflecting, in part, the lesser sensitivity of this measure.

To see if the pattern of impairment noted in the first 10 trials was characteristic of the overall performance of the groups, additional contrasts were made on both mean latency and the mean number of failures to respond across all $\mathbf{4 0}$ trials. No significant differences were found between the control groups on either measure, and these two were again combined for further comparisons with the shocked groups. Group AC-C, AC-P, and DC-C each had longer mean block latencies, $p<.001$, and failed to respond on more trials, $\mathrm{p}<.05$, than did the controls, thus reflecting a persistence of the interference with escape-avoidance responding. The difference between DC-P and the controls continued to be small and nonsignificant. While there was some improvement over blocks for all groups (see Figures 2 and 3), the pattern established in the first 10 test trials was largely maintained.

\section{Discussion}

Group AC-C received an inescapable shock treatment which was quite similar to Overmier and Seligman's (1967) primary "helplessness treatment"; the impairment found in these two groups was also 
very similar. They reported that $63 \%$ of their subjects never responded during the 10 test trials, while $67 \%$ of the subjects in our AC-C never responded during the first 10 trials. Similar levels of the impairment were found in our Groups AC-P $(67 \%)$ and DC-C $(50 \%)$, but Group DC-P (17\%) performed at a level which resembled that of the control groups $(17 \%)$. These findings both extend and place limitations on the generality of the "learned helplessness" hypothesis. Also, the results reported by Anderson et al. (Note 1) in rats using dc inescapable shock have been replicated here in dogs. Dogs receiving the DC-C treatment showed significantly lower activity ratings to the inescapable shocks than those receiving the DC-P treatment. During testing, the DC-C group showed persistently impaired escape-avoidance responding across test trials, while the DC-P group showed no such impairment. Although activity patterns for groups that received ac shocks were similar to their dc counterparts (i.e., high for pulsating shock and low for continuous shock), their test results were not. Both groups that received ac inescapable shocks were substantially impaired throughout testing.

\section{EXPERIMENT 2}

This study was undertaken at Notre Dame University laboratories to determine whether the pattern of results found in Experiment 1 applied as well to rats. The same experimental design was used. Slight modifications were made in the parameters and procedures so that they would conform closely to those conditions used by Anderson et al. (Note 1). The most notable changes included the administration of three daily sessions of inescapable shock prior to four consecutive daily escape-avoidance test sessions of 12 trials each.

\footnotetext{
Method

Subjects and Apparatus

The subjects, 36 male albino Sprague-Dawley rats, 90 to 100 days of age at the start of the experiment, were maintained in separate cages and given free access to food and water throughout the experiment. They were handled and weighed daily and acclimated to the laboratory for 7 to 10 days prior to experimentation.

As in Experiment 1, separate and distinctly different units were used for each phase of the experiment. A flat platform composed of clear acrylic plastic, elevated on three wooden dowels over a painted plywood base, was employed for restraining subjects during the presentation of inescapable shock. The platform provided separate relief areas for the rat's genitalia, head, and limbs. Each subject was lightly anesthetized (20- to 40-sec exposure to either in a closed jar) and was strapped onto the plastic platform by securing an elastic bandage around the torso and by taping the forelimbs together to the front dowel and the hindlimbs separately to the respective adjacent rear dowels. A polished copper-penny electrode was lightly moistened with electrode paste and firmly attached to the outstretched sole of each hind paw with adhesive tape.
}

The shock source used to supply dc shock was the same as that described in Experiment 1. The ac shock source was one modeled after Campbell and Teghtsoonian (1958). The shock level was set at $4 \mathrm{~mA}$ for the inescapable shock phase. For groups receiving pulsating shock, a pulse-stream generator interrupted the shock at a rate of 10 pulses $/ \mathrm{sec}$ with equal on-off intervals.

The motion detector described in Experiment 1 was again used to record gross bodily movements during the inescapable shock presentations. The transmitting and receiving transducers of the device were located $9 \mathrm{~cm}$ apart center to center and supported on rods $17 \mathrm{~cm}$ from the subject's right side, with the receiving transducers perpendicular to the head and shoulder alignment of the subject. The sensitivity adjustment was set at 1.0 , which readily detected all visible gross body movements. A sound-deadened, lightcontrolled, ventilated refrigerator shell was used to house all restrained subjects. All treatment contingencies were controlled with solid state equipment and a laboratory computer housed in another room.

An $11.3 \mathrm{~cm}$ wide $\times 59.7 \mathrm{~cm}$ long $\times 25.0 \mathrm{~cm}$ high (inside) shuttlebox was employed for testing. The shuttlebox had a grid floor composed of grids, $.32 \mathrm{~cm}$ in diameter and $1.27 \mathrm{~cm}$ apart center to center, stainless steel walls that were connected to the grid circuit, and an electrifiable cylindrical hurdle $(5.1 \mathrm{~cm}$ diam) mounted on ball bearings across the center of the apparatus. The hurdle extended $6.3 \mathrm{~cm}$ above the floor and easily rotated when touched, thereby preventing the subject from perching on it. The onset of two $71 / 2-W$ lamps served as the CS and were located $22 \mathrm{~cm}$ above the floor at either end of the chamber. The grid floor was divided in half and switches were attached to each floor assembly. A full crossing from one compartment to the other was required in order to activate the switches and be defined as a response. The shock source for the shuttlebox test was the scrambled $40-\mathrm{V}$ ac output of a transformer connected in a series with a $22-k \Omega$ resistor. A computer recorded response latencies and intertrial crossings, and controlled all stimulus presentation and temporal contingencies.

\section{Procedure}

Inescapable shock treatment. After being secured on the plastic platform with the electrodes in place, each subject was given $6 \mathrm{~min}$ to adapt to the apparatus before the shock treatment began. On each of 3 consecutive days, Groups AC-C, AC-P, DC-C, and DC-P each received 645 -sec unsignaled shocks programmed to be inescapable. The ISI ranged from 45 to $75 \mathrm{sec}$ with an average of $60 \mathrm{sec}$. The shocks were from either ac or dc shock sources and were either continuous or pulsating, forming the four groups, AC-C, AC-P, DC-C, and DC-P. Group NS was only restrained for an equal period of time, and Group NT received no treatment prior to testing in the shuttlebox.

Testing for interference. All subjects were given escapeavoidance training approximately $24 \mathrm{~h}$ after the last day of inescapable shock treatment. The escape-avoidance trials followed the method of emergence described in Experiment 1. Following a 2-min adaptation period to the apparatus, 12 escape-avoidance trials were run with a CS-US interval of $5 \mathrm{sec}$ and a variable ITI of $60 \mathrm{sec}( \pm 30 \mathrm{sec})$. This procedure was repeated on each of 3 additional days for a total of 4 test days in all. Latency to respond after CS onset was recorded. If no response occurred within $60 \mathrm{sec}$ of CS onset, the trial was automatically terminated and a latency of $60 \mathrm{sec}$ was recorded.

\section{Activity Data}

\section{Results}

Figure 4 shows the mean activity rate across blocks of eight shock presentations for each inescapable shock group. The overall mean activity values for Groups AC-C, AC-P, DC-C, and DC-P, were 8.9, $13.8,3.7$, and 13.1 , respectively. The temporal form 


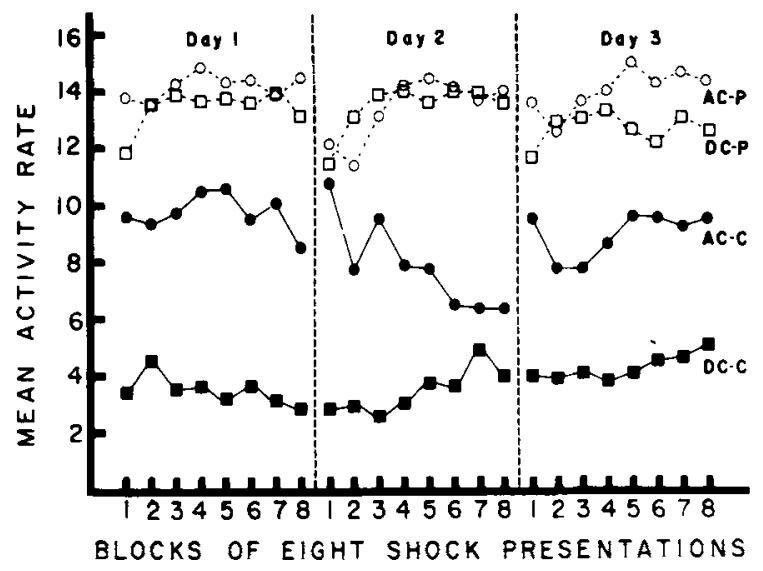

Figure 4. Mean activity rates are shown across blocks of eight inescapable shock presentations on each of three daily treatment sessions for each experimental group in Experiment 2. Group treatments differed with respect to shock source (i.e., AC or DC) and temporal form of shock (i.e., Continuous or Pulsating). Data are presented for six rats in each group.

of the shock again had a marked effect on activity, with pulsating groups moving more than continuous shock groups. An ANOVA was performed on the individual mean activity counts across the eight eighttrial blocks of shock over the 3 days (Source by Form by Days by Blocks). This revealed the activity differences due to shock form to be significant, $F(1,20)=176.16, p<.001$. Shock source was again an influential factor, with ac groups having higher activity rates than the dc groups, $F(1,20)=29.36$, $\mathrm{p}<.001$. There was a significant Source by Form interaction, $F(1,20)=17.55, \mathrm{p}<.001$.

Pairwise comparisons between shock treatment groups revealed that neither the two pulsated groups nor the two continuous shock groups differed significantly from each other, $\mathrm{t}(20)=.21$ and 1.62 , both ps $>.05$. The large difference in activity rates between the dc shock groups reached significance, $t(20)=2.95, p<.01$, but the lesser difference between the two ac shock groups did not, $t(20)=1.53$, $\mathrm{p}>.05$.

This pattern of high activity from the two pulsating shock groups, low activity from Group DC-C and intermediate level from Group AC-C was relatively stable over successive daily exposures, as Days proved not to be a significant factor. However, there was sufficient change across blocks to reveal a significant Source by Form by Blocks interaction within days, $\mathrm{F}(21,420)=2.59, \mathrm{p}<.001$. The fluctuations across blocks were rarely sustained in one direction, but rather appeared as unsystematic rises and falls in activity (see Figure 4).

\section{Test Data}

The treatment of the test data was the same as that described in Experiment 1 except that 12-trial blocks (i.e., days) were used. The first 12 trials, constituting the first day of testing, were separately analyzed, followed by an analysis over all blocks.

As might well be expected, given the available literature on shuttle avoidance in rats, latencies proved to be a substantially more sensitive measure of impairment than did the number of failures to respond. No significant differences were found between the combined shock groups and the controls for the number of failures to escape on either the first 12 test trials or over all 48 test trials. Consequently, the remainder of the analyses are based on latency data alone.

Figure 5 shows the mean daily latencies for each of the shock treatment and control groups. Although the restrained control group, NS, did have a somewhat longer mean latency than the no-treatment group, NT, on the first 12 trials, this difference was not significant, $p>.05$. These two groups were therefore combined for purposes of contrasts with the shocked experimental groups. The mean latency of the four combined shock groups on the first test day (Block 1) was significantly longer than that of the combined controls, $p<.001$. This pattern of the interference on Block 1 was again influenced by both shock source and temporal form of the inescapable shock, with these factors interacting, $p<.05$. As was true for the dogs, not all shock treatments were effective in producing impairment on the first day of testing; only Groups AC-C, AC-P, and DC-C were found to have significantly longer mean latencies than the combined controls, $\mathrm{p}<.001, \mathrm{p}<.001$, and $p<.05$, respectively. Differences among these three impaired groups were not significant. The mean

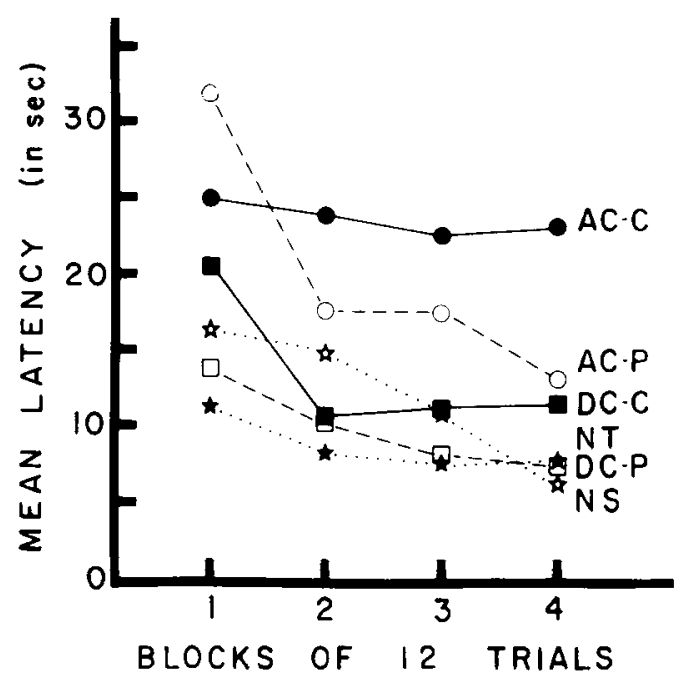

Figure 5. Mean shuttle response latency from CS onset for each group in Experiment 2 across blocks of 12 signaled escapeavoidance trials. Each block of trials constitutes one daily test session. Groups differed with respect to the inescapable shock treatment received prior to test. See text for further explanation. 
latency of Group DC-P was between those of the two control groups and was not significantly different from them. Of the three impaired groups, only AC-P differed significantly from DC-P, $\mathrm{p}<.05$.

The distribution of treatment groups between the "impaired" and "not impaired" categories on the first block of testing is identical to that found in the dogs for their first block trials. Although this pattern was persistent throughout testing in the dogs, it was not maintained here. The combined shock groups still performed more poorly than did the controls when the overall mean latencies of all four blocks were analyzed, $p>.05$, but only Groups AC-C and AC-P remained significantly slower than the controls, both $\mathrm{p}<.01$. Group DC-C, however, improved over days (see Figure 5) and failed to have a significantly longer mean latency over all blocks than did controls, $p>.05$. Group DC-P continued to not differ from controls, $\mathrm{p}>.05$. These findings suggest that inescapable shock source is an especially influential factor in determining the pattern of impairment over days for the rats tested here.

\section{Discussion}

As was the case in the previous study with dogs, both groups receiving pulsating shocks showed relatively higher activity rates during shock than the corresponding continuous shock groups. On Day 1 of testing, groups that had received ac pulsating and continuous shocks both showed impairment, as did the group that received inescapable dc continuous shock. But the group that had received dc pulsating shocks showed no impairment. This replicated Anderson's et al. (Note 1) earlier findings, when they compared dc continuous and pulsating shock treatments, and extends the comparisons to include ac shock treatment.

However, the pattern during Day 1 of testing was not maintained. While the AC-C and AC-P groups continued to show longer latencies than did the controls, the DC-C group showed decreasing latencies across the test days (see Figure 5), such that their performance came to resemble that of the control animals. This is to be contrasted with the earlier findings of Anderson et al. (Note 1) in which the impairment found in dc continuous shock group was somewhat more sustained.

\section{GENERAL DISCUSSION}

The present studies confirm and extend the earlier reports of Overmier and Seligman (1967) and Anderson et al. (Note 1), and reveal that the differences obtained in those experiments are not simply attributable to differences in species. Indeed, there are striking similarities between the initial patterns of results in Experiments 1 and 2, suggesting that the interference phenomenon is sensitive to similar parameters in both dogs and rats. Inescapable ac shock resulted in substantially and persistently impaired test performances of both dogs and rats regardless of the temporal form of the inescapable shock. This lack of differential effect due to temporal form of the ac shock on test performances was obtained in spite of the large differential effect it had on prior activity rates in the dogs.

In contrast, the differential effect produced by the temporal form of inescapable dc shock was evident in both activity levels and test performances by dogs and rats. The dc continuous shock produced low activity rates during inescapable shocks, and later resulted in impaired escape-avoidance performance. In rats, however, this impairment was transient; in dogs, the impairment more closely resembled that observed in the ac shock groups.

Clearly, an interaction between shock source and temporal form was evident. The potential for such interactions among shock parameters is hardly a new issue, yet it is one that has not been thoroughly considered in the literature on the effects of inescapable shock upon subsequent (escape-avoidance) responding. This issue is becoming increasingly important as certain shock parameters come to play more key roles in the proposed explanations of the observed interference effects; for example, Glazer and Weiss (1976) have emphasized both the duration and intensity of inescapable shock in their analysis of the observed interference.

In trying to identify those properties of the inescapable shock responsible for observed differences in escape-avoidance performance, it may be important to note the relative similarity between the inescapable shock and the shock used in the escapeavoidance task. While source and form of the inescapable shock were systematically varied in both experiments, the traditional ac shock source delivering scrambled grid shock was always used in the test phase. A recent experiment by Rosellini and Seligman (in press) indicates that similarity of the inescapable and training shocks is an important variable. They found that exposure of rats to inescapable shocks impaired subsequent escape behavior only if the inescapable and the escapable shocks were similar in intensity. Interpretation of our data in terms of "similarity" requires that on some basis one arrange the four types of inescapable shock with respect to their similarities to each other and to the scrambled grid shock used in the escape/avoidance phase. One might choose as a basis some descriptive property of the shocks or even their effective intensity (i.e., severity), using as an independent measure of severity the activity levels elicited in Phase 1. The descriptive property which scales the treatments in terms of the obtained interference is not intuitively obvious here, and we already have noted that the activity patterns do not "predict" the amount of interference to be 
observed. This is not to deny that similarity may account for our results; it is just that the dimensionality of such similarity is yet to be identified. Finally, we ought to note that while similarity of the inducing and testing conditions may well modulate the interference phenomenon, it is not necessary. Altenor, Kay, and Richter (1977) showed that similarity is not necessary by demonstrating that inescapable submersion in water is effective in producing interference with a shock escape task in rats while inescapable shock is effective in interfering with a water escape task.

Most of the current hypotheses offered to explain the interference with escape-avoidance responding have not addressed themselves to the role of the different shock sources and temporal forms, and none of them can account easily for the total pattern of results found here. The "learned helplessness" hypothesis which places critical emphasis on the uncontrollability of shock cannot readily account for the differential impairment in groups here. Inescapability of the shock is not per se a sufficient condition for insuring subsequent interference with escapeavoidance responding. The present experiments built upon the series of experiments reviewed by Maier and Seligman (1976), which made clear, via the triadic design, that only inescapable shocks produced interference; those experiments used AC-C inducing shocks. But, as the present experiments directly raise questions about the sufficiency of inescapability, they also indirectly raise questions about its necessity under the other inducing shock treatments as well. Is there a critical interaction between escapability, shock parameters, and their necessity and sufficiency in producing interference?

The competing response hypotheses offered by Bracewell and Black (1974), Weiss, Kriekhaus, and Conte (1968), and Anderson et al. (Note 1) suggest that a direct relationship exists between activity during the inescapable shock and subsequent escapeavoidance performance. However, while the activity rates for the AC-P and DC-P groups were virtually indistinguishable, their test performances were quite dissimilar. In addition, while the activity rates of the AC-P and AC-C groups were dissimilar (though not statistically so for the rats), interference was obtained under both conditions. Clearly, no simple relationship between gross activity levels during inescapable shocks and later escape-avoidance responding can be postulated that holds for all shock treatments in these experiments.

It remains for future investigations to elucidate the special properties of the inescapable shocks used here to account for the dissimilarities in test performance. This must be done if we are to have a viable account of the interference phenomenon. The current results caution against attempting any precipitous conclusions about the role of shock source and temporal form variables until we achieve a better understanding of their interactions with other shock parameters already known to influence the interference phenomenon, such as duration and both absolute and relative intensity (Glazer \& Weiss, 1976; Rosellini \& Seligman, in press).

\section{REFERENCE NOTE}

1. Anderson, D. C., Lupo, V., Cunningham, C.. \& Madden, J. Variations in pretreatments as determinants of shutlebox behavior and pain elicited aggression. Paper presented in the 15th Annual Meeting of the Psychonomic Society. Boston, 1974.

\section{REFERENCES}

Altenor, A., Kay, E.. \& Riceter, M. The generality of learned helplessness in the rat. Leaming and Motivation. 1977. 8. 54-61.

Bracewell, R. J.. \& Black, A. H. The effects of restraint and noncontingent pre-shock on subsequent escape learning in the rat. Learning and Motivation, 1974, 5. 53-69.

Campbell. B. A., \& Teghtsoonian. R. Electrical and behavioral effects of different types of shock stimuli on the rat. Joumal of Comparative and Physiological Psychology. 1958. 51, 185-192.

D'Amato. M. R., \& Fazzaro. J. Discriminated lever-press avoidance learning as a function of type and intensity of shock. Journal of Comparative and Physiological Psychology, 1966. 61. 313-315.

Edgington. E. S. Statistical inference: The distribution-free approach. New York: McGraw-Hill, 1969. Chapter S. (a)

Edgington. E. S. Approximate randomization tests. Journal of Psychologi. 1969, 72, 143-149. (b)

Fisher. R. A. The design of experiments. Edinburgh: Oliver \& Boyd. 1935

Follick, M. J., \& KNutson, J. F. Shock source and intensity: Variables in shock-induced fighting. Behavior Research Methods \& Instrumentation, 1974, 6. 477-480.

Freda. J. S., \& KLein, S. B. Generality of the failure-to-escape (helplessness) phenomenon in rats. Animal Learning \& Behavior. 1976. 4. $401-406$.

Glazer, H. L.. \& Weiss. J. M. Long-term and transitory interference effects. Joumal of Experimental Psychology: Animal Behavior Processes, 1976. 2, 191-201.

Maier. S. F.. \& Seligman, M. E. P. Learned helplessness: Theory and evidence. Journal of Experimental Psychology: General. 1976, 105, 3-46.

Maler. S. F.. Seligman, M. E. P.. \& Solomon, R. L. Pavlovian tear conditioning and learned helplessness. In B. A. Campbell \& R. M. Church (Eds.), Punishment. New York: Appleton-Century-Crotts, 1969.

Nash, R. F., \& Gallup, G. G. Effect of different parameters of shock on tonic immobility. Behavior Research Methods \& Instrumentation, 1975, 7. 361-364.

Overmier. J. B., \& Seligman. M. E. P. Etfects of inescapable shock upon subsequent escape and avoidance responding. Journal of Comparative and Physiological Psychology, 1967, 63, 28-33.

Rosellini, R. A., \& Seligman, M. E. P. Role of shock intensity in the learned helplessness paradigm. Animal Leaming \& Behavior, 1978, 6, 143-146.

Solomon, R. L., \& BRUse, E. S. Experimentally derived conceptions of anxiety and aversion. In $M$. R. Jones (Ed.), Nebraska symposium on motivation, 1956. 35. 212-305.

Weiss. J. M.. Krieckhaus. E. E.. \& Conte. R. Effects of fear conditioning on subsequent avoidance behavior and movement. Journal of Comparative and Physiological Psychology. 1968. 65. 413-421.

(Received for publication May 12. 1977 revision accepted September 25, 1977.) 